\title{
El concepto de tratamiento all-on-four en el tratamiento de pacientes con prótesis fija de arcada completa
}

The all-on-four Treatment Concept in the Treatment of Patients with a Complete-Arch Fixed Prosthesis

Alejandro Aldana Caceres ${ }^{1 \mathrm{ac}}$, Jaime Barcena Taco ${ }^{1 \mathrm{bc}}$

(1) https://orcid.org/0000-0001-7758-1411 (10) https://orcid.org/0000-0003-3082-3369

\section{Resumen}

El objetivo de esta revisión es analizar el tratamiento de pacientes edéntulos a través de prótesis fijas sobre cuatro implantes colocados el mismo día de la intervención bajo el concepto del tratamiento all-on-four. Se revisa ejes centrales como el manejo de la técnica, planificación y evaluación. Finalmente, se muestran resultados en los que se aplican la técnica en mención, obtenidos a través de la búsqueda automatizada en la base de datos PubMED, SCOPUS, SciELO, Cochrane y NGC. A través de la revisión de artículos publicados, se determinó que la utilización de implantes inclinados mejoró el desempeño clínico protésico al favorecer la distribución poligonal de los implantes, minimizar el cantiléver, evitar tratamientos de injertos óseos, minimizar la morbilidad quirúrgica, disminuir los tiempos y costos.

\section{Palabras clave: all-on-four, pacientes edéntulos}

\begin{abstract}
The objective of this review is to analyze the treatment of edentulous patients through fixed prostheses on four implants placed on the same day of the intervention under the concept of all-on-four treatment. Central axes such as technique management, planning and evaluation are reviewed. Finally, results are shown in which the aforementioned technique is applied, obtained through the automated search in the PubMED, SCOPUS, SciELO, Cochrane and NGC databases. Through the review of published articles, it was determined that the use of inclined implants improved prosthetic clinical performance by favoring the polygonal distribution of implants, minimizing cantilever, avoiding bone graft treatments, minimizing surgical morbidity, reducing times and costs.
\end{abstract}

Keywords: all-on-four, edentulous patients

\section{Introducción}

Los injertos óseos tipo onlay y la elevación de la membrana sinusal son opciones a tratamientos de maxilares severamente reabsorbidos pero podrían tener baja aceptación por los pacientes por el riesgo de morbilidad y altos costos. ${ }^{1}$

La instalación de implantes inclinados son una opción que permiten sortear la neumatización del seno maxilar o una

\footnotetext{
${ }^{1}$ Escuela Profesional de Odontología, Universidad Nacional Jorge Basadre Grohmann, Tacna, Perú

${ }^{a}$ Doctor en Ciencias de la Salud

${ }^{\mathrm{b}}$ Magíster en Docencia Universitaria y Gestión Educativa

${ }^{\mathrm{c}}$ Docente
} 
reabsorción severa de la mandíbula $a^{2,3}$ incrementándose la posibilidad de colocar implantes de mayor longitud y mejorar la distribución poligonal de soporte protésico sin necesidad de recurrir a un injerto óseo o relleno de seno maxilar. ${ }^{4,5}$

El concepto de tratamiento all-on-four existe desde hace casi veinte años. Durante este tiempo, se utilizó para tratar a miles de pacientes totalmente edéntulos y potencialmente edéntulos. Esta alternativa ofrece ventajas únicas frente a las convencionales, tanto para el paciente como para el clínico.

En 1998, el Dr. Paulo Malo trató con éxito al primer paciente con el concepto de tratamiento all-on-four. Desde entonces, se ha tratado a cientos de miles de pacientes. ${ }^{6}$

El concepto de tratamiento all-on four (all-on-4), ofrece a los pacientes edéntulos y potencialmente edéntulos una prótesis de arcada completa fija sobre cuatro implantes el mismo día de la intervención recuperando la función, estética, percepción, habla y autoestima. ${ }^{7,8}$

\section{Implicancias del concepto all-on-four}

1. Restauración de arcada completa con solo cuatro implantes: dos implantes anteriores rectos y dos implantes posteriores inclinados hasta $45^{\circ}$.

2. Función inmediata (puente acrílico fijo) para los pacientes que cumplan los criterios para carga inmediata de los implantes.

3. Procedimiento sin injertos.

4. Dos aspectos clave reducen considerablemente la complejidad de tratamiento: el número de intervenciones y el tiempo total del tratamiento. Al inclinar los implantes posteriores, se evita la necesidad de realizar procedimientos de injerto de hueso, el cual requieren mucho tiempo. La carga inmediata, con una prótesis provisional fija acorta el tiempo hasta conseguir dientes funcionales. ${ }^{7}$

5. El concepto de tratamiento all-on-four no solo es la opción de tratamiento que menos tiempo requiere, sino también la menos costosa en comparación con métodos convencionales de tratamiento con implantes para pacientes edéntulos o potencialmente edéntulos que utilizan cirugía en dos fases. ${ }^{9}$

6. Si comparamos grupos dentados y el concepto all-on-four, ambos presentan un patrón de contracción similar a la electromiografía de la superficie muscular (sEMG) lo que significa que no hay diferencia estadística entre ambos grupos. ${ }^{10,11,12}$

\section{Planificación del tratamiento}

Como parte de la evaluación sistemática del paciente, pueden ser útiles las siguientes acciones:

1. Revisar la presencia o falta de tejido duro o blando puede ayudar a determinar el tipo de prótesis definitiva.

2. Identificar una línea de transición puede ayudar a determinar posibles consideraciones y necesidades estéticas. ${ }^{13}$.

3. Verificar que la línea de transición sea apical a la línea de la sonrisa, lo que produce un resultado estético.

4. Evitar un resultado antiestético en tanto la línea de transición es coronal a la línea de la sonrisa.

5. Evaluar las zonas del maxilar puede ayudar a seleccionar un protocolo de tratamiento quirúrgico y restaurador específico.

6. Es recomendable pensar en utilizar software 3D para evaluar las posibles ubicaciones para la colocación de los implantes.

EI Dr. Jivraj, un destacado prostodoncista de Estados Unidos y experto en el concepto de tratamiento all-on-four, sugiere los siete factores de diagnóstico siguientes para ayudar a determinar si el paciente es un candidato adecuado para una restauración fija o removible: El borde incisal, espacio para la restauración, soporte labial, línea de la sonrisa, contornos, superficie de los tejidos y la oclusión. 


\section{Tratamiento bajo el concepto de all-on- four}

El tratamiento del maxilar requiere la evaluación del hueso alveolar disponible. Los pacientes con hueso solo en la zona I y la zona II podrían ser candidatos para el concepto de tratamiento all-on-four.

Aunque es posible tener un defecto solo de dientes en la mandíbula edéntula, la mayoría de los pacientes presentan algún grado de reabsorción ósea. Las opciones de tratamiento quirúrgico para este grupo de pacientes incluyen implantes colocados axialmente o inclinados para soportar un puente fijo de implantes o una sobredentadura fija - removible sobre barra de implantes. ${ }^{6}$

En la mandíbula, la inclinación de los implantes distales puede prevenir daño al nervio mentoniano y dentario inferior. En el maxilar superior, el uso de implantes dentales inclinados son una alternativa a procedimientos de injerto óseo y elevación de seno maxilar. ${ }^{14}$ Implantes de longitud convencional se pueden colocar inclinados permitiendo el uso de mayor cantidad posible de hueso cortical, lo cual aumenta estabilidad primaria. $^{15}$

Además, aumentar la distancia entre implantes y reducir la longitud del voladizo permite una mejor distribución de carga. ${ }^{16}$ Varios estudios computarizados revelan ventajas en la biomecánica de la inclinación del implante en restauraciones de arco completo. ${ }^{15,17}$

\section{Consideraciones sobre la planificación del tratamiento}

El concepto de tratamiento all-on-four se ha desarrollado con el objetivo de maximizar el hueso disponible y permitir una función inmediata. Durante la planificación de un tratamiento utilizando una técnica con colgajo se debe considerar lo siguiente:

- Capacidad de lograr suficiente estabilidad primaria del implante para carga inmediata.

- Los implantes deben soportar un torque mínimo de $35 \mathrm{Ncm}$.

- Si no se logra el torque, se recomienda una fase de cicatrización convencional antes de suministrar la restauración provisional o definitiva.

- Volumen de hueso disponible: indicado para maxilares edéntulos con una anchura ósea mínima de $5 \mathrm{~mm}$ y una altura ósea mínima de $10 \mathrm{~mm}$ de canino a canino.

- Volumen de hueso disponible: indicado para mandíbulas edéntulas con una anchura ósea mínima de $5 \mathrm{~mm}$ y una altura ósea mínima de $8 \mathrm{~mm}$ entre los forámenes mentonianos.

- Se aconseja colocar los implantes entre los alvéolos postexodoncia.

- Para reducir los cantilevers, incline los implantes posteriores a un máximo de $45^{\circ}$.

- En el caso de implantes posteriores inclinados, planifique los orificios de acceso a los tornillos distales de modo que queden situados a la altura del plano oclusal del primer molar, el segundo premolar o el primer premolar.

Las inserciones de los implantes inclinados pueden conducir a una mayor reducción de los valores de estrés en la interfaz hueso-implante en comparación con los implantes no inclinados. ${ }^{16}$

La literatura actual muestra que la carga inmediata de implantes dentales presenta una alta tasa de éxito de supervivencia del implante. Es una alternativa adecuada para la mayoría de pacientes que necesitan rehabilitación total de la mandíbula. ${ }^{18}$

La carga inmediata asociada con la inclinación implantes es una modalidad de tratamiento válida para el maxilar atrófico. ${ }^{19}$

En un estudio retrospectivo que incluyó 176 implantes cargados inmediatamente con prótesis fijas en mandíbulas edéntulas a través del concepto all-on-four, mostraron una resorción ósea marginal mínima en todos los implantes. ${ }^{20}$

La pérdida de hueso marginal alrededor los implantes inclinados entablillados para sostener una prótesis fija no fueron significativamente diferentes en comparación a implantes rectos. ${ }^{21}$ Así mismo, la inclinación del implante no influye 
significativamente en la resorción ósea periimplantaria después de cinco años de carga. ${ }^{22}$

En un metanálisis reciente, no se encontró diferencias significativas entre los implantes dentales inclinados en la ocurrencia de una mayor pérdida ósea marginal en comparación con los implantes colocados axialmente. ${ }^{23}$ No hay diferencias significativas entre las tasas de éxito de los implantes axiales y los inclinados.

Por otra parte, la pérdida de hueso marginal fue similar para los implantes inclinados y axiales en los estudios prospectivos y retrospectivos sometidos a revisión. Los implantes inclinados exhiben el mismo comportamiento que los implantes colocados en posición axial.

\section{Conclusiones}

La utilización de implantes inclinados mejora el desempeño clínico protésico favoreciendo la distribución poligonal de los implantes, minimizando el cantiléver, evitando tratamientos de injertos óseos, minimizando la morbilidad quirúrgica y disminuyendo tiempos y costes del tratamiento.

No hay diferencia significativa en la actividad electromiográfica comparando los implantes dentales colocados bajo el concepto de all-on-four y los implantes axiales.

\section{Referencias}

1. Wallace SS, Froum SJ. Effect of maxillary sinus augmentation on the survival of endosseous dental implants as compared to the survival of implants placed in the non-grafted posterior maxilla: An evidence-based literature review. Ann Periodontol, Dic 2003; 8: 328-343. Doi: 10.1902/annals.2003.8.1.328.

2. Krekmanov L. Placement of posterior mandibular and maxillary implants in patients with severe bone deficiency: A clinical report of procedure. Int J Oral Max Impl, Sept-oct 2000; 15: 722-730. PMID: 11055139.

3. Krekmanov L, Kahn M, Rangert B, Lindström $\mathrm{H}$. Tilting of posterior mandibular and maxillary implants for improved prosthesis support. Int J Oral
Max Impl, May-jun 2000; 15: 405414.PMID: 10874806.

4. Fortin Y, Sullivan RM, Rangert B. The Marius implant bridge: Surgical and prosthetic rehabilitation for the completely edentulous upper jaw with moderate to severe resorption: A 5-year retrospective clinical study. Clin Implant Dent R, 2002; 4(2): 69-77. Doi: $10.1111 /$ j.17088208.2002.tb00155.x.

5. Calandriello R, Tomatis M. Simplified treatment of the atrophic posterior maxilla via immediate/early function and tilted implants: A prospective 1-year clinical study. Clin Implant Dent Relat Res. 2005;7 Suppl 1:S1-12. Doi: 10.1111/j.17088208.2005.tb00069.x.

6. Nobel Biocare TM [Libro electrónico]. Concepto de tratamiento All-on-4, 2016. Disponible en: shorturl.at/enyF8

7. Data Research U.S. and European Markets for Overdentures, 2014.

8. Maló P, Rangert B, Nobre M. All-on-4 immediate-function concept with Brånemark System implants for completely edentulous maxillae: a 1-year retrospective clinical study. Clin Implant Dent Relat Res. 2005; 7 Suppl 1:S88-94. Doi: 10.1111/j.1708-8208.2005.tb00080.x.

9. Babbush CA. Posttreatment quantification of patient experiences with full-arch implant treatment using a modification of the OHIP-14 questionnaire. J Oral Implantol. Jun 2012; 38(3):251-60. Doi: 10.1563/AAID-JOI-D-12-00001.

10. De Rossi M, Santos CM, Migliorança R, Regalo SC. All on Four ${ }^{\circledR}$ fixed implant support rehabilitation: a masticatory function study. Clin Implant Dent Relat Res. Agto 2014; 16(4):594-600. Doi: 10.1111/cid.12031.

11. Dellavia C. Electromyographic assessment of jaw muscles in patients with All-on-Four fixed implan-tsupported prostheses. J Oral Rehabil 2012 Dec; 39(12):896-904. Dellavia C, Francetti L, Rosati R, Corbella S, Ferrario VF, Sforza C. Electromyographic assessment of jaw muscles in patients with All-on-Four fixed implant-supported prostheses. J Oral Rehabil. Dic 2012; 39(12):896-904. Doi: 10.1111/joor. 12002.

12. Tartaglia GM, Testori T, PallaveraA, Marelli $B$, Sforza C. Electromyographic analysis 
of masticatory and neck muscles in subjects with natural dentition, teethsupported and implant-supported prostheses. Clin Oral Implants Res. Oct 2008; 19(10):1081-8. Doi: 10.1111/j.16000501.2008.01574.x.

13. Bedrossian E, Sullivan RM, Fortin $Y$, Malo $\mathrm{P}$, Indresano T. Fixed-prosthetic implant restoration of the edentulous maxilla: a systematic pretreatment evaluation method. J Oral Maxillofac Surg. Jan 2008; $66(1): 112-22$. D o i : 10.1016/j.joms.2007.06.687.

14. Aparicio C, Perales P, Rangert B. Tilted implants as an alternative to maxillary sinus grafting: a clinical, radiologic, and periotest study. Clin Implant Dent Relat Res. $2001 ; 3(1): 39-49$. Doi : 10.1111/j.1708-8208.2001.tb00127.x.

15. Zampelis A, Rangert B, Heijl L. Tilting of splinted implants for improved prosthodontic support: a two-dimensional finite element analysis. J Prosthet Dent. Jun 2007; 97(6 Suppl):S35-43. Doi: 10.1016/S0022-3913(07)60006-7. Erratum in: J Prosthet Dent. Mar 2008; 99(3):167.

16. Bellini CM, Romeo D, Galbusera F, Taschieri S, Raimondi MT, Zampelis A, Francetti L. Comparison of tilted versus nontilted implant-supported prosthetic designs for the restoration of the edentuous mandible: a biomechanical study. Int J Oral Maxillofac Implants. Mayjun 2009;24 (3):511-7. PMID: 19587875.

17. Bellini CM, Romeo D, Galbusera F, Agliardi E, Pietrabissa R, Zampelis A, Francetti $L$. A finite element analysis of tilted versus nontilted implant configurations in the edentulous maxilla. Int J Prosthodont. Mar-Apr 2009; 22(2):155-7. PMID: 19418861.

18. Del Fabbro, M.; Testori, T.; Francetti, L.; Weinstein, R.L. Systematic review of survival rates for implants placed in grafted maxillary sinus. Int. J. Periodontics Restor. Dent. 2004, 24 (6), 565-577. Doi: 10.1016/j.prosdent.2005.04.024
19. Testori T, Del Fabbro M, Capelli M, Zuffetti F, Francetti L, Weinstein RL. Immediate occlusal loading and tilted implants for the rehabilitation of the atrophic edentulous maxilla: 1-year interim results of a multicenter prospective study. Clin Oral Implants Res. Mar 2008;19(3):227-32. Doi: 10.1111/j.1600-0501.2007.01472.x.

20. Maló P, Rangert B, Nobre M. "All-on-Four" immediate-function concept with Brånemark System implants for completely edentulous mandibles: a retrospective clinical study. Clin Implant Dent Relat Res. 2003; 5 Suppl 1:2-9. Doi: 10.1111/j.1708-8208.2003.tb00010.x. PMID: 12691645.

21. Monje A, Chan HL, Suarez F, GalindoMoreno P, Wang HL. Marginal bone loss around tilted implants in comparison to straight implants: a meta-analysis. Int $\mathrm{J}$ Oral Maxillofac Implants. Nov-dic 2012; 27(6):1576-83. PMID: 23189313

22. Koutouzis T, Wennström JL. Bone level changes at axial- and non-axial-positioned implants supporting fixed partial dentures. A 5-year retrospective longitudinal study. Clin Oral Implants Res. Oct 2007; 18(5):585-90. doi: $10.1111 / \mathrm{j} .1600-$ 0501.2007.01386.x.

23. Chercanovic, B.R.; Albrektsson, T.; Wennenberg, A. Tilted versus axially placed dental implants: A meta-analysis. J. Dent. 2015, 2, 149-170. [CrossRef] [PubMed]

24. Ata-Ali J, Peñarrocha-Oltra D, CandelMarti E, Peñarrocha-Diago M. Oral rehabilitation with tilted dental implants: a metaanalysis. Med Oral Patol Oral Cir Bucal. 1 jul 2012;17(4):e582-7. doi: 10.4317/medoral.17674.

25. Leighton $Y$, Von Marttens A. Immediate prosthetic function with All on-4 mandibular technique: First report after 1 year of immediate loading. Rev. Clin. Periodoncia Implantol. Rehabil Oral. Abr 2014; 7(1):32-36. Doi: 10.4067/S071901072014000100008.
Correspondencia:

aaldanac@unjbg.edu.pe
Fecha de recepción : 20 de julio de 2020

Fecha de aceptación : 15 de septiembre de 2020 